PROCEEDINGS OF THE

AMERICAN MATHEMATICAL SOCIETY

Volume 130, Number 6, Pages 1563-1572

S 0002-9939(01)06241-4

Article electronically published on October 24, 2001

\title{
OCTONION ALGEBRAS OBTAINED FROM ASSOCIATIVE ALGEBRAS WITH INVOLUTION
}

\author{
HOLGER P. PETERSSON AND MICHEL L. RACINE
}

(Communicated by Lance W. Small)

\begin{abstract}
A natural octonion algebra structure on the symmetric elements of trace 0 of central simple associative algebras of degree 3 with involution of the second kind is obtained.
\end{abstract}

\section{INTRODUCTION}

Given a central simple associative algebra of degree 3 with involution of the second kind $(B, *)$, several constructions in the literature can be viewed as associating to it an octonion algebra. Our main concern in this paper is to derive an explicit formula for the multiplication of an octonion algebra living on the space of symmetric trace 0 elements of $(B, *)$ and to relate it to previous constructions.

The first construction is due independently to Okubo [6] and Faulkner [2] and yields an eight-dimensional (nonunital) symmetric composition algebra on the elements of trace 0 of a central simple associative algebra $A$ of degree 3 , provided that the base field has characteristic not 2 or 3 and contains the cube roots of unity. Since, by passing to a principal Albert isotope (see, for example, 1]), every eightdimensional composition algebra determines a unique octonion algebra having the same norm, the Okubo-Faulkner construction, in a roundabout way, produces an octonion algebra structure on the elements of trace 0 in $A$.

Next there is a construction due to Haile, Knus, Rost and Tignol 3 attaching to any central simple associative algebra $(B, *)$ of degree 3 with involution of the second kind a 3-fold Pfister form which reflects important cohomological invariants of algebraic groups and, in particular, classifies the involutions of the second kind on a fixed algebra $B$. We refer to [5, Chapter V] for a systematic account of the construction. Since 3-fold Pfister forms are exactly the norm forms of octonion algebras and since an octonion algebra is determined up to isomorphism by its norm form, the Haile-Knus-Rost-Tignol construction again produces an octonion algebra structure, this time determined in a roundabout manner by $(B, *)$.

Our purpose is to make this octonion algebra structure explicit. Rather than working within the narrow confines of $(B, *)$ itself, we prefer to enlarge it to a reduced Albert algebra $J$ by means of the Tits process [7, 8], allowing us, via the explicit coordinatization obtained in 11, to identify the pure octonions of the coordinate algebra of $J$ with certain symmetric elements of trace 0 in $(B, *)$. An

Received by the editors July 19, 2000 and, in revised form, November 22, 2000.

2000 Mathematics Subject Classification. Primary 17A75, 16W10, 17C40.

The second author's research was supported in part by a grant from NSERC. 
analogous approach had previously been adopted for the coordinate norm 9], 10, leading to an alternate treatment of the 3 -fold Pfister form attached to $(B, *)$ (see also [5, Chapter 40] for related results). But while the latter approach works in all characteristics, here we have to exclude characteristics 2 and 3. Finally, we specialize $(B, *)$ to $A+A^{o p}$ equipped with the exchange involution, $A$ being a central simple associative algebra of degree 3 , and in this way relate our octonion algebra structure to the symmetric composition algebra obtained by Okubo and Faulkner.

\section{Algebras of Degree 3}

We start by recalling facts concerning algebras of degree 3 . Let $(B, *)$ be a central simple associative algebra with involution over a field $k$. Then $B$ is either simple or the direct sum $A \oplus A^{o p}$, where $A$ is simple and $A^{o p}$ denotes the opposite algebra, i.e., $a^{o p} b:=b a$; in this case $*$ is the exchange involution which permutes the summands. Assume that $*$ is of the second kind, i.e., is not the identity on the centre of $B$. Then $K$, the centre of $B$, is either a separable quadratic field extension of $k$ or $K=k \oplus k$. Let $H(B, *)=\left\{b \in B \mid b^{*}=b\right\}$, the symmetric elements of $B$. If $B=A \oplus A^{o p}$, then $H(B, *)=\{(a, a) \mid a \in A\}$ which can be identified with $A$, at least as a vector space. We will also identify $K$ with $K 1 \subseteq B$.

If $B$ is of degree 3 over its centre, then every element satisfies its reduced characteristic polynomial

$$
x^{3}-T(x) x^{2}+S(x) x-N(x),
$$

where $T$ is the reduced trace, $N$ the reduced norm and $S$ a quadratic form, which we refer to as the quadratic trace. The forms $T, S$ and $N$ on all of $B$ take values in $K$ but their restrictions to $H(B, *)$ take values in $k$. The quadratic trace $S(x)$ is given by

$$
S(x):=T\left(x^{\#}\right),
$$

where ${ }^{\#}$ is the adjoint, i.e., the numerator of the inversion map:

$$
x^{\#}=x^{2}-T(x) x+S(x) .
$$

Recall that for $x \times y:=(x+y)^{\#}-x^{\#}-y^{\#}$,

$$
1 \times y=T(y)-y .
$$

Let

$$
T(a, b):=T(a b) .
$$

From now on assume that $k=H(K, *)$ is of characteristic not 2 or 3 . In this case, combining (2) and (3),

$$
S(x)=\frac{1}{2}\left(T(x)^{2}-T\left(x^{2}\right)\right) .
$$

We wish to obtain more precise information on the behaviour of the quadratic form $S$ on $H(B, *)_{0}$, the symmetric elements of trace 0 . For example, (5) becomes

$$
S(x)=-\frac{1}{2} T\left(x^{2}\right), \quad x \in H(B, *)_{0} .
$$

The linearization $S(x, y)=S(x+y)-S(x)-S(y)$ becomes

$$
S(x, y)=-T(x, y), \quad x, y \in H(B, *)_{0} .
$$


From $x^{\# \#}=N(x) x$ and $S\left(x^{\#}\right)=T\left(x^{\# \#}\right)=N(x) T(x)$, we deduce

$$
S\left(x^{\#}\right)=0, \quad x \in H(B, *)_{0} .
$$

Moreover

$$
S\left(x^{2}, 1\right)=T\left(x^{2} \times 1\right)=T\left(T\left(x^{2}\right)-x^{2}\right)=2 T\left(x^{2}\right) .
$$

So, by $\left(5^{\prime}\right)$,

$$
\begin{aligned}
S\left(x^{2}, 1\right) & =-4 S(x), \\
S\left(x^{\#}\right) & =S\left(x^{2}+S(x)\right) \\
& =S\left(x^{2}\right)+S(x) S\left(x^{2}, 1\right)+S(x)^{2} S(1) \\
& =S\left(x^{2}\right)-4 S(x)^{2}+3 S(x)^{2}
\end{aligned}
$$

and, by (6),

$$
S\left(x^{2}\right)=S(x)^{2}, \quad x \in H(B, *)_{0} .
$$

Since the characteristic is not 2 , there exists an element $\theta \in K$ such that $K=$ $k[\theta], \theta^{*}=-\theta$ and $\theta^{2}=\rho \in k$. Recall that a frame of $B$ is a set of pairwise orthogonal primitive idempotents whose sum is the unit element of $B$. We will need the existence of certain symmetric elements of $B$.

Lemma 1. If $(B, *)$ is a central simple associative algebra of degree 3 with involution of the second kind, then there exists a $d \in H(B, *)$ satisfying

$$
T(d)=0 \quad \text { and } \quad S(d) \neq 0 \neq N(d) .
$$

Proof. If $B$ is a division algebra or $B=A+A^{o p}$, with $A$ a division algebra, then the norm form is anisotropic on $H(B, *)$. Since $H(B, *)=k+H(B, *)_{0}$ is an orthogonal splitting with respect to the trace bilinear form $T(x, y)$ and, by $\left(5^{\prime \prime}\right)$, $S(x, y)=-T(x, y)$ on $H(B, *)_{0}, S(x)$ is nonsingular on $H(B, *)_{0}$ and we may choose a $d \in H(B, *)_{0}$ with $S(d) \neq 0 \neq N(d)$. On the other hand, if $H(B, *)$ contains a frame $\left\{e_{1}, e_{2}, e_{3}\right\}$, one checks that $d=e_{1}+e_{2}-2 e_{3}$ satisfies $T(d)=0$, $S(d)=-3$ and $N(d)=-2$. So in all cases there exists a $d \in H(B, *)$ satisfying $T(d)=0$ and $S(d) \neq 0 \neq N(d)$.

Remark. If the base field is infinite, such d's are Zariski dense.

We now recall a few facts concerning exceptional simple Jordan algebras or Albert algebras, as they are now known [4, Chapter IX], 7], 8]. Albert algebras are of degree 3 over their centre and thus satisfy equation (1) for $S(x)$ and $x^{\#}$ as in equations (2) and (3). In fact the structure of an Albert algebra is determined by its cubic norm form $N$ and the associated trace form $T$ and adjoint \#. Linearizing \#,

$$
x \times y:=(x+y)^{\#}-x^{\#}-y^{\#},
$$

and comparing with (3)

$$
x \times y=x \circ y-T(x) y-T(y) x+S(x, y)
$$

and, in characteristic not 2 , the Jordan product $x \cdot y=\frac{1}{2} x \circ y$, while in general the quadratic Jordan algebra structure is given by

$$
y U_{x}:=T\left(x^{\#}, y\right) x-x^{\#} \times y .
$$


We denote by $U_{x, z}$ the linearization of $U_{x}$, i.e.,

$$
y U_{x, z}:=y U_{x+z}-y U_{x}-y U_{z} .
$$

Every Albert algebra $J$, central over the field $k$, can be obtained from a central simple associative algebra $(B, *)$ of degree 3 with involution of the second kind, using the Tits process as follows. For $u \in H(B, *)$ and $\mu \in K$ invertible such that $N(u)=\mu \mu^{*}$,

$$
J=J(B, *, u, \mu):=H(B, *) \oplus B,
$$

as a vector space. For $(a, b),(r, s) \in J(B, *, u, \mu)$, one defines an adjoint \#, a norm form $N$ and a trace form $T$ on $J$ which extend those on $H(B, *)$,

$$
\begin{aligned}
(a, b)^{\#} & :=\left(a^{\#}-b u b^{*}, \mu^{*} b^{* \#} u^{-1}-a b\right), \\
N((a, b)) & :=N(a)+\mu N(b)+\mu^{*} N\left(b^{*}\right)-T\left(a b u b^{*}\right), \\
T((a, b)) & :=T(a) .
\end{aligned}
$$

Moreover

$$
\begin{aligned}
S((a, b)) & =T\left((a, b)^{\#}\right)=T\left(a^{\#}-b u b^{*}\right) \\
& =S(a)-T\left(b u b^{*}\right), \\
(a, b) \times(r, s) & =\left(a \times r-b u s^{*}-s u b^{*}, \mu^{*}\left(b^{*} \times s^{*}\right) u^{-1}-a s-r b\right) .
\end{aligned}
$$

Since, for $J(B, *, u, \mu)$ to be a division algebra, it is necessary that $\mu \notin N(B)$ 7, Th. 5.2], $J(B, *, 1,1)$ is not a division algebra. So it is reduced and contains a frame. We will construct an explicit one. Let

$$
e_{1}=\frac{1}{3}(1,1) \quad \text { and } \quad f=1-e_{1}=\frac{1}{3}(2,-1) .
$$

Since $T\left(e_{1}\right)=1$ and $e_{1}^{\#}=0$, using (2) and (3) we have $0=e_{1}^{2}-e_{1}$ and $e_{1}$ is a primitive idempotent of $J$. One also checks that

$$
f^{\#}=e_{1} \text {. }
$$

For $d$ as in Lemma 1, let

$$
c=\frac{1}{2} S(d)^{-1} d^{2}+\frac{1}{3} .
$$

By $\left(5^{\prime}\right), T(c)=0$ and $c \in H(B, *)_{0}$. We will also need

$$
\begin{aligned}
S(c) & =\frac{1}{4} S(d)^{-2} S\left(d^{2}\right)+\frac{1}{6} S(d)^{-1} S\left(d^{2}, 1\right)+\frac{1}{3} \\
& =\frac{1}{4}-\frac{2}{3} S(d)^{-1} S(d)+\frac{1}{3} \\
& =-\frac{1}{12} .
\end{aligned}
$$

So, for $c$ as in (16), we have

$$
T(c)=0 \quad \text { and } \quad S(c)=-\frac{1}{12} .
$$


For arbitrary $(a, b) \in J$, we have

$$
\begin{aligned}
e_{1} \times(a, b) & =\frac{1}{3}\left(T(a)-a-b-b^{*}, T\left(b^{*}\right)-a-b-b^{*}\right), \\
(2,-1) \times(a, b) & =\left(2 T(a)-2 a+b^{*}+b,-T\left(b^{*}\right)+b^{*}-2 b+a\right), \\
(c, c) \times(a, b) & =\left(a \times c-c b^{*}-b c, c \times b^{*}-c b-a c\right), \\
(c, c)^{\#} & =\left(c^{\#}-c^{2}, c^{\#}-c^{2}\right)=-\left(\frac{1}{12}, \frac{1}{12}\right) .
\end{aligned}
$$

We will use the Peirce decomposition of $J$ with respect to an idempotent $e$. Recall [4. p.120] that the Peirce 1, 0 and $\frac{1}{2}$ spaces of a unital Jordan algebra are given by

$$
J_{1}(e)=J U_{e}, \quad J_{0}(e)=J U_{1-e}, \quad J_{\frac{1}{2}}(e)=J U_{e, 1-e} .
$$

Moreover, in an Albert algebra $J$, [11, equations (28), (29)] for a primitive idempotent $e$ and an arbitrary element of $J$,

$$
\begin{aligned}
& T(x)=0 \quad \text { and } \quad e \times x=0 \quad \Longleftrightarrow x \in J_{\frac{1}{2}}(e), \\
& T(x)=0 \quad \text { and } \quad e \times x=-x \quad \Rightarrow x \in J_{0}(e) .
\end{aligned}
$$

Lemma 2. If $(B, *)$ is a central simple associative algebra of degree 3 with involution of the second kind, let d be chosen as in Lemma 1 and $c$ as in equation (16). Then

$$
e_{1}=\frac{1}{3}(1,1), \quad e_{2}=\left(\frac{1}{3}+c,-\frac{1}{6}+c\right) \quad \text { and } \quad e_{3}=\left(\frac{1}{3}-c,-\frac{1}{6}-c\right)
$$

form a frame of $J(B, *, 1,1)$.

Proof. Since $e_{2}=\frac{1}{2} f+(c, c)$ and $e_{3}=\frac{1}{2} f-(c, c)$, we have $T\left(e_{i}\right)=1, e_{1}+e_{2}+e_{3}=1$ and, by (19) and (21),

$$
\begin{aligned}
e_{2}^{\#} & =\frac{1}{4} f^{\#}+\frac{1}{2} f \times(c, c)+(c, c)^{\#} \\
& =\frac{1}{12}(1,1)-\left(\frac{1}{12}, \frac{1}{12}\right)=(0,0) .
\end{aligned}
$$

Similarly $e_{3}^{\#}=(0,0)$ and we have a system of primitive idempotents. Now $T((c, c))$ $=T(c)=0$ and, by (18), $e_{1} \times(c, c)=-(c, c)$. So, by $(23),(c, c) \in J_{0}\left(e_{1}\right)$. Since $f \in J_{0}\left(e_{1}\right)$, by (24), $e_{2}$ and $e_{3}$ are also elements of $J_{0}\left(e_{1}\right)$. Thus $e_{1}$ is orthogonal to $e_{2}$ and $e_{3}$ and since $e_{2}$ is a primitive idempotent of $J U_{f}=J_{0}\left(e_{1}\right), f-e_{2}=e_{3}$ is orthogonal to $e_{2}$ and we have a frame of $J$.

\section{An OCTONION STRUCTURE ON $H(B, *)_{0}$}

Let $(B, *)$ be a central simple associative algebra of degree 3 with involution of the second kind over a field $k$ of characteristic not 2 or 3 . Write $K=k(\theta)$ $\left(\theta^{*}=-\theta, \theta^{2}=\rho, \rho \in k\right)$ for the centre of $B$. For an element $g \in H(B, *)_{0}$, denote by $g^{\perp}$ the orthogonal complement of $g$ with respect to $T$. We write $[a, b]=a b-b a$, $a \circ b=a b+b a$.

Let $\mathcal{C}$ be an octonion algebra over $k$ with norm $n$ and trace $t$. Since char $k \neq 2$, $\mathcal{C}=k+\mathcal{C}_{0}, \mathcal{C}_{0}=\{a \in \mathcal{C} \mid t(a)=0\}$, and the multiplication of $\mathcal{C}$ is determined by the product of pure octonions, i.e., elements of $\mathcal{C}_{0}$. 
Theorem. If $(B, *)$ is a central simple associative algebra of degree 3 with involution of the second kind over a field $k$ of characteristic not 2 or 3 , let $d$ be a fixed element of $H(B, *)_{0}$ with $S(d) \neq 0 \neq N(d)$. For arbitrary elements a, $b \in H(B, *)_{0} \cap d^{2 \perp}$, the product

$$
a \cdot b:=18 \rho T(a, b) S(d)^{2} w
$$

$$
\begin{aligned}
& +3 \theta\left(-3[a, b] \circ d^{2}-3 a d^{2} b+3 b d^{2} a+2 T(b, d)[a, d]\right. \\
& \left.-2 T(a, d)[b, d]-4 S(d)[a, b]+2 T([a, b], d) d+T\left([a, b], d^{2}\right)\right),
\end{aligned}
$$

where $w$ is a formal unit element, endows $H(B, *)_{0} \cap d^{2 \perp}$ with a pure octonion structure with unit element $w$ and norm form

$$
n(a):=36 \rho S(d)^{2} S(a) .
$$

Proof. We will prove this by embedding $H(B, *)_{0} \cap d^{2 \perp}$ in the reduced Albert algebra $J=J(B, *, 1,1)$ and then using the explicit coordinatization obtained in 11. Recall that this is done by choosing a frame, fixing a Peirce space, say $J_{23}=J U_{e_{2}, e_{3}}$, choosing elements $u \in J_{12}$ and $v \in J_{31}$ with $S(u) \neq 0 \neq S(v)$ and defining a product on $J_{23}$ as

$$
a \cdot b:=(v \times a) \times(u \times b), \quad a, b \in J_{23} .
$$

This endows $J_{23}$ with an octonion algebra structure whose norm and unit element are

$$
\begin{aligned}
n(a) & :=-S(u) S(v) S(a), \quad a \in J_{23}, \\
1: & =(S(u) S(v))^{-1} u \times v .
\end{aligned}
$$

Let $J=k e_{1}+k e_{2}+k e_{3}+J_{12}+J_{23}+J_{31}$ be the Peirce decomposition of $J$ with respect to the frame $e_{1}, e_{2}, e_{3}$ as in Lemma 2. By (18) and (12), $H(B, *)_{0}$ embeds in $\left\{(a, a) \mid a \in H(B, *)_{0}\right\}$ an 8-dimensional subspace of $J_{0}\left(e_{1}\right)$. By (19) and (20), for $a \in H(B, *)_{0}$,

$$
\begin{aligned}
f \times(a, a) & =(0,0), \\
(c, c) \times(a, a) & =-T(a, c)(1,1) .
\end{aligned}
$$

So, by (22), $\left\{(a, a) \mid a \in H(B, *)_{0}, T(a, c)=0\right\}$ is a 7-dimensional subspace of $J_{23}$. For $a \in H(B, *)_{0}, T(a, c)=\frac{1}{2} S(d)^{-1} T\left(a, d^{2}\right)$ and $T(a, c)=0$ if and only if $T\left(a, d^{2}\right)=0$. By $(18), e_{1} \times(0, \theta)=-(0, \theta)$ and $(0, \theta) \in J_{0}\left(e_{1}\right)$. By (19) and (20),

$$
\begin{gathered}
\frac{1}{6}(2,-1) \times(0, \theta)=(0,0) \\
(c, c) \times(0, \theta)=(\theta c-c \theta,-\theta 1 \times c-\theta c)=(0,0)
\end{gathered}
$$

and $(0, \theta) \in J_{23}$. So

$$
J_{23}=k(0, \theta) \oplus\left\{(a, a) \mid a \in H(B, *)_{0}, T(a, c)=0\right\} .
$$

Moreover $S((0, \theta))=T\left(\theta^{2}\right)=3 \rho \neq 0$.

To define an octonion structure on $J_{23}$ we need not determine $J_{12}$ and $J_{31}$ but need only find an element of each of these spaces whose quadratic trace is nonzero. 
For this we will need

$$
\begin{aligned}
c d & =\frac{1}{2} S(d)^{-1} d^{3}+\frac{1}{3} d=\frac{1}{2} S(d)^{-1}(-S(d) d+N(d))+\frac{1}{3} d \\
& =\frac{1}{2} S(d)^{-1} N(d)-\frac{1}{6} d
\end{aligned}
$$

and

$$
S(c, d)=-T(c, d)=-T(c d)=-\frac{3}{2} S(d)^{-1} N(d) .
$$

Let $u=(0, \theta d)$. Using (19), (20), (30) and (31), we have $f \times u=-u,(c, c) \times u=$ $(0,-\theta(c \times d+c d))=\left(0,-\theta(3 c d+S(c, d))=\frac{1}{2} u\right.$. So $e_{3} \times u=-u, e_{2} \times u=0$ and $u \in J_{12}$. Moreover $S(u)=T\left(u^{\#}\right)=T\left(\theta^{2} d^{2}\right)=-2 \rho S(d) \neq 0$. Let $v=$ $(2 d,-d)$. Again using (19), (20), (30) and (31), we have $f \times v=-v,(c, c) \times v=$ $(6 c d+2 S(c, d),-3 c d-S(c, d))=-\frac{1}{2} v$. Hence $e_{2} \times v=-v, e_{3} \times v=0$ and $v \in J_{31}$. Also $S((2 d,-d))=T\left(4 d^{\#}-d^{2}\right)=4 S(d)+2 S(d)=6 S(d)$ and $u \times v=2 S(d)(0, \theta)$.

If $x, y \in J_{23}$,

$$
x \cdot y:=((2 d,-d) \times x) \times((0, \theta d) \times y)
$$

defines an octonion stucture on $J_{23}$ with norm form

$$
n(x):=12 \rho S(d)^{2} S(x)
$$

and unit element

$$
(S(u) S(v))^{-1} u \times v=(-2 \rho S(d) 6 S(d))^{-1} 2 S(d)(0, \theta)=-\frac{1}{6} \rho^{-1} S(d)^{-1}(0, \theta) .
$$

By $(13)$ and $\left(5^{\prime}\right)$, for $a \in H(B, *)_{0}, S((a, a))=3 S(a)$. So for $a \in H(B, *)_{0} \cap d^{2 \perp}$,

$$
n(a)=36 \rho S(d)^{2} S(a) .
$$

Using $\left(10^{\prime}\right),(0, \theta 1) \times(a, a)=(0,0)$ and the decomposition in (29) is orthogonal with respect to $S$ and hence with respect to $n$. Since the octonion unit element is a scalar multiple of $(0, \theta 1)$, the pure octonions, i.e., the octonions of trace 0 , correspond to $H(B, *)_{0} \cap d^{2 \perp}$.

For $a, b \in H(B, *)_{0} \cap d^{2 \perp}$,

$$
\begin{aligned}
(2 d,-d) \times(a, a) & =(2 a \times d+d a+a d,-a \times d-2 d a+a d) \\
& =(3 a \circ d-2 T(a, d), T(a, d)-3 d a)
\end{aligned}
$$

while

$$
\begin{aligned}
(0, \theta d) \times(b, b) & =(-\theta d b+b \theta d,-\theta b \times d-b \theta d) \\
& =(\theta[b, d], \theta(T(b, d)-2 b d-d b)) .
\end{aligned}
$$

If we cross the two expressions above, we get

$$
\begin{aligned}
((2 d, & -d) \times(a, a)) \times((0, \theta d) \times(b, b)) \\
= & \theta((3 a \circ d-2 T(a, d)) \times[b, d]+(T(a, d)-3 d a)(T(b, d)-2 d b-b d) \\
& -(T(b, d)-2 b d-d b)(T(a, d)-3 a d) \\
& -(T(a, d)-3 a d) \times(T(b, d)-2 d b-b d) \\
& -(3 a \circ d-2 T(a, d))(T(b, d)-2 b d-d b)-[b, d](T(a, d)-3 d a)) .
\end{aligned}
$$


Expanding the first component of (32), we get

$$
\begin{gathered}
3 \theta((a \circ d) \circ[b, d]+d[a, b] d+2[d a d, b] \\
+T(b, d)[a, d]-T(a, d)[b, d]-T(a \circ d,[b, d])) \\
=3 \theta\left([a, d b d]-[b, d a d]+2 d[a, b] d-a d^{2} b+b d^{2} a\right. \\
\left.+T(b, d)[a, d]-T(a, d)[b, d]-T\left([a, b], d^{2}\right)\right),
\end{gathered}
$$

since the trace is invariant under cyclic permutations. In a Jordan algebra of degree 3 ,

$$
x z x=z U_{x}=T(z, x) x-x^{\#} \times z .
$$

For an element $z$ of trace 0 ,

$$
\begin{aligned}
d z d & =T(z, d) d-d^{\#} \circ z+S(d) z-S\left(z, d^{\#}\right) \\
& =-z \circ d^{2}-S(d) z+T(z, d) d+T\left(z, d^{2}\right) .
\end{aligned}
$$

Using this, the above expression can be transformed into

$$
\begin{aligned}
3 \theta & \left(-3[a, b] \circ d^{2}-3 a d^{2} b+3 b d^{2} a+2 T(b, d)[a, d]\right. \\
& \left.-2 T(a, d)[b, d]-4 S(d)[a, b]+2 T([a, b], d) d+T\left([a, b], d^{2}\right)\right) .
\end{aligned}
$$

This is the pure octonion component of the product. We could obtain the appropriate multiple of the unit element by considering the second component of (32) but we choose to use the norm form. In an octonion algebra $x=\frac{1}{2} t(x) 1+x_{0}=$ $\frac{1}{2} n(x, 1) 1+x_{0}$, where $x_{0}$ is of trace 0 . By $\left(27^{\prime}\right), n(a b, 1)=n(a, \bar{b})=-n(a, b)=$ $-36 \rho S(d)^{2} S(a, b)$ and the coefficient of the unit element in the product of $a, b$ is $-18 \rho S(d)^{2} S(a, b)$.

Corollary 1. Up to isomorphism, the octonion algebra structure on $H(B, *)$ obtained above is independent of the choice of $d$. Moreover if $K$ is split, then so is the corresponding octonion algebra.

Proof. Choosing a different $d$ may yield a different frame on $J(B, *, 1,1)$. However the coordinate algebras relative to different frames are isomorphic by the AlbertJacobson Theorem [4, chapter IX, Theorem 1].

If $K$ is split, then $B=A \oplus A^{o p}$ and $J(B, *, 1,1)$ is a first Tits construction [7, Theorem 3.5] which must be split since it is reduced [4, chapter IX, Theorem $20]$.

Corollary 2. Every octonion algebra $C$ over $k$ arises from the construction of the Theorem.

Proof. Write $J=H_{3}(C)$ for the Albert algebra of 3-by-3 hermitian matrices having entries in $C$ and consider any central simple associative algebra $(B, *)$ of degree 3 with involution of the second kind such that $H(B, *)$ is a subalgebra of $J$ (e.g., $B=M_{3}(K)$, the algebra of 3-by-3 matrices having entries in a two-dimensional étale subalgebra $K \subset C, *$ being the conjugate transpose involution). Then there are invertible elements $u \in H(B, *), \mu \in K$ (the centre of $B$ ) satisfying $N(u)=\mu \mu^{*}$ such that $J \cong J(B, *, u, \mu)$ [8] Theorem 3.1]. Since $J$ is reduced, $\mu=N(y)$ for some $y \in B^{\times}$[7, Theorem 5.2], allowing us to assume $\mu=1$ [7, Proposition 3.7]. 
But then, by 77, Proposition 3.8], some isotope $J^{(v)}$ of $J$ becomes isomorphic to $J(B, *(u), 1,1), *(u)$ being the $u$-twist $x \mapsto u x^{*} u^{-1}$ of $*$. Since the coordinate algebra of $J^{(v)}$ continues to be isomorphic to $C$, by the Albert-Jacobson Theorem [4, Chapter IX, Theorem 1], $C$ is isomorphic to the octonion algebra of $(B, *(u))$ constructed in the proof of the Theorem.

Remarks. 1. The converse of the last statement of Corollary 1 is false since a second Tits construction can also be a first Tits construction [8, Theorem 5.2].

2. As pointed out by Faulkner [2], the octonion algebra arising from his construction is split. Since, in our case, it corresponds to $K$ split, so is the octonion algebra of our Theorem.

3. The isometry class of the norm form obtained above corresponds to the mod 2 invariant $f_{3}(J(B, *, 1,1))$ of $J(B, *, 1,1)$ [5 $\left.\S 40\right],[9$.

4. As was noted in the proof of the Theorem, $H(B, *)_{0} \cap d^{2 \perp}=H(B, *)_{0} \cap c^{\perp}$. By (17), $c \in H(B, *)_{0}$ so $H(B, *)_{0}=k c \oplus H(B, *)_{0} \cap d^{2 \perp}$ and the two summands are orthogonal with respect to the quadratic form $S$. We could let a multiple of $c$ act as the identity for our octonion structure on $H(B, *)_{0}$. However, by (17), $36 \rho S(d)^{2} S\left(S(d)^{-1} c\right)=-3 \rho$ and this will never be more than an artificial choice.

5. A composition algebra is symmetric if the associated bilinear form is associative. Symmetric composition algebras are the subject of [5, §34]. Theorem 34.37 (3) of [5] can be paraphrased to say that symmetric composition algebras of dimension 8 are obtained from simple associative algebras with involution of the second kind of degree 3 over their centre and from octonion algebras. In view of Corollary 2, our Theorem may be construed as saying that all symmetric composition algebras of dimension 8 are obtained from simple associative algebras with involution of the second kind of degree 3 over their centre.

Let us finally recall the construction of Haile, Knus, Rost and Tignol [3, $5 \S 19$. B]. Denote by $\left\langle\left\langle\alpha_{1}, \alpha_{2}, \ldots, \alpha_{n}\right\rangle\right\rangle$ the $n$-fold Pfister form

$$
\left\langle 1,-\alpha_{1}\right\rangle \otimes\left\langle 1,-\alpha_{2}\right\rangle \otimes \cdots \otimes\left\langle 1,-\alpha_{n}\right\rangle .
$$

On $H(B, *)$ as above, the quadratic form

$$
Q_{*}(x):=T\left(x^{2}\right) \simeq\langle 1,1,1\rangle \perp\langle 2\rangle \cdot\langle\langle\rho\rangle\rangle \cdot q_{*},
$$

where $q_{*}$ is a 3 -dimensional quadratic form of determinant 1 . Since $q_{*}$ is of determinant 1 , the form $\pi_{*}=\langle\langle\rho\rangle\rangle \cdot q_{*} \perp\langle\langle\rho\rangle\rangle$ is a 3 -fold Pfister form which determines the involution * of $B$ up to conjugacy [5, Theorem 19.6] and yields a cohomological invariant $f_{3}(B, *)$ [5, Theorem 30.21].

Proposition. Let $(B, *)$ be a central simple associative algebra of degree 3 over $K$ with involution of the second kind, $K=k(\theta), \theta^{*}=-\theta, \theta^{2}=\rho, k$ a field of characteristic not 2 or 3 and $n$ the unique octonion norm form corresponding to $(B, *)$. The 3 -fold Pfister forms $\pi_{*}$ and $n$ are isometric and hence the corresponding octonion algebras are isomorphic.

Proof. By [5, Theorem 40.2, (2)], the invariant $f_{3}(J(B, *, 1,1))$ is the $f_{3}$-invariant of the involution $*$ of $B$.

Remark. Since the $f_{3}$-invariant of the involution $*$ of $B$ is an invariant of $(B, *)$ this provides another proof of Corollary 1. 


\section{REFERENCES}

1. Elduque, A. and Pérez, J.M., Composition algebras with associative bilinear forms, Comm. Algebra 24 (1996), 1091-1116. MR 97b:17006

2. Faulkner, J., Finding octonion algebras in associative algebras, Proc. Amer. Math. Soc. 104 (1988), 1027-1030. MR 89g:17003

3. Haile, D., Knus, M.-A., Rost, M. and Tignol, J.-P., Algebras of odd degree with involution, trace forms and dihedral extensions, Israel J. Math. 96 (1996), 299-340. MR 98h:16024

4. Jacobson, N., Structure and Representations of Jordan Algebras, AMS Colloquium Publications Vol. 39, 1968, Providence R.I. MR 40:4330

5. Knus, M.-A., Merkurjev, A., Rost, M. and Tignol, J.-P., The Book of Involutions, American Mathematical Society, Providence R.I., 1998, American Mathematical Society Colloquium Publications Vol. 44. MR 2000a:16031

6. Okubo, S., Pseudo-quaternion and pseudo-octonion algebras, Hadronic J. 1 (1978), 1250-1278. MR 80a: 17004

7. Petersson, H.P., and Racine, M.L., Jordan algebras of degree 3 and the Tits process, J. Algebra 98 (1986), 211-243. MR 87h:17038a

8. Petersson, H.P., and Racine, M.L., Classification of algebras arising from the Tits process, J. Algebra 98 (1986), 244-279. MR 87h:17038b

9. Petersson, H.P., and Racine, M.L., On the invariants mod 2 of Albert algebras, Journal of Algebra 174 (1995) 1049-1072. MR 96c:17045

10. Petersson, H.P., and Racine, M.L., Reduced models of Albert algebras, Math. Zeitschrift 223 (1996) 367-385. MR 98c:17028

11. Racine, M.L., A note on quadratic Jordan algebras of degree 3, Trans. Amer. Math. Soc., 164 (1972), 93-103. MR 46:3582

Fachbereich Mathematik, FernUniversitaet, D-58084 Hagen, Germany

E-mail address: holger.petersson@fernuni-hagen.de

Department of Mathematics \& Statistics, University of Ottawa, Ottawa, Ontario, CANADA K1N 6N5

E-mail address: mracine@uottawa.ca 